\title{
F0X01 Suppression Is a Determinant of Acquired Lapatinib-Resistance in HER2-Positive Gastric Cancer Cells through MET Upregulation
}

\author{
Jinju Park, MS ${ }^{1}$ \\ Yiseul Choi, MS ${ }^{1}$ \\ Young San Ko, PhD2 \\ Younghoon Kim, $\mathrm{MD}^{3}$ \\ Jung-Soo Pyo, MD, PhD ${ }^{4}$ \\ Bo Gun Jang, MD, $P h D^{5}$ \\ Min A Kim, MD, PhD ${ }^{3}$ \\ Jae-Seon Lee, $\mathrm{PhD}^{6}$ \\ Mee Soo Chang, MD, $\mathrm{PhD}^{3}$ \\ Jong-Wan Park, MD, PhD \\ Byung Lan Lee, MD, PhD 1,7,8
}

\begin{abstract}
${ }^{1}$ Tumour Biology, Cancer Research Institute, Seoul National University College of Medicine, Seoul, ${ }^{2}$ Department of Forensic Medicine, National Forensic Service Busan Institute, Yangsan, ${ }^{3}$ Department of Pathology, Seoul National University College of Medicine, Seoul, ${ }^{4}$ Department of Pathology, Eulji University Hospital, Eulji University School of Medicine, Daejeon,

${ }^{5}$ Department of Pathology, Jeju National University Hospital, Jeju, ${ }^{6}$ Department of Molecular Medicine, Inha University College of Medicine, Incheon, Ischemic/Hypoxic Disease Institute Medical Research Center, Seoul National University College of Medicine, Seoul, ${ }^{8}$ Department of Anatomy, Seoul National University College of Medicine, Seoul, Korea
\end{abstract}

Correspondence: Byung Lan Lee, MD, PhD Department of Anatomy, Seoul National University College of Medicine, 103 Daehak-ro, Jongno-gu, Seoul 03080, Korea

Tel: $82-2-740-8218$

Fax: 82-2-745-9528

E-mail: dslanat@snu.ac.kr

Received December 7, 2016

Accepted March 22, 2017

Published Online March 24, 2017

\section{Purpose}

Lapatinib is a candidate drug for treatment of trastuzumab-resistant, human epidermal growth factor receptor 2 (HER2)-positive gastric cancer (GC). Unfortunately, lapatinib resistance renders this drug ineffective. The present study investigated the implication of forkhead box 01 (FOX01) signaling in the acquired lapatinib resistance in HER2-positive GC cells.

\section{Materials and Methods}

Lapatinib-resistant GC cell lines (SNU-216 LR2-8) were generated in vitro by chronic exposure of lapatinib-sensitive, HER2-positive SNU-216 cells to lapatinib. SNU-216 LR cells with FOXO1 overexpression were generated by stable transfection of a constitutively active FOXO1 mutant (FOX01A3). HER2 and MET in SNU-216 LR cells were downregulated using RNA interference. The sensitivity of GC cells to lapatinib and/or cisplatin was determined by crystal violet assay. In addition, Western blot analysis, luciferase reporter assay and reverse transcription-polymerase chain reaction were performed.

\section{Results}

SNU-216 LR cells showed upregulations of HER2 and MET, but downregulation of FOXO1 compared to parental SNU-216 cells. FOXO1 overexpression in SNU-216 LR cells significantly suppressed resistance to lapatinib and/or cisplatin. In addition, FOXO1 negatively controlled HER2 and MET at the transcriptional level and was negatively controlled by these molecules at the post-transcriptional level. A positive crosstalk was shown between HER2 and MET, each of which increased resistance to lapatinib and/or cisplatin.

\section{Conclusion}

FOXO1 serves as an important linker between HER2 and MET signaling pathways through negative crosstalks and is a key regulator of the acquired lapatinib resistance in HER2-positive GC cells. These findings provide a rationale for establishing a novel treatment strategy to overcome lapatinib resistance in a subtype of GC patients.

\section{Key words}

Stomach neoplasms, ErbB-2 receptor, Drug resistance, Lapatinib, Human FOXO1 protein, Human MET protein 


\section{Introduction}

Gastric cancer (GC) constitutes a major cause of cancerrelated deaths worldwide, but its management, especially in advanced stages, has evolved relatively little. Human epidermal growth factor receptor 2 (HER2/ ErbB2/ neu) is a 185$\mathrm{kDa}$ transmembrane receptor tyrosine kinase (RTK) and a member of the epidermal growth factor receptor (EGFR) family [1]. HER2 serves as an important therapeutic target for therapy in HER2-positive metastatic GC since its overexpression is found in more than 15\% of GC and is associated with poor prognosis, particularly in the advanced stages of disease [2]. For patients with HER2-positive advanced GC, trastuzumab (Herceptin, Genentech) combined with standard chemotherapy has been used as first-line treatment $[2,3]$. However, intrinsic and/or acquired resistance totrastuzumab became a major obstacle in anti-HER2 therapy for advanced GC [2]. Thus, there is a need for alternatives to block HER2 signaling in GC.

Lapatinib (Tykerb, GlaxoSmithKline, Ware, UK) is an oral dual tyrosine kinase inhibitor of EGFR (HER1) and HER2 [4]. Although lapatinib could be used for treatment of trastuzumab-resistant, HER2-positive advanced GC cases, the major problem of therapies targeting tyrosine kinases is that many tumors do not response or eventually develop resistance to the drugs, often due to activation of alternative signaling pathways [5]. Therefore, it is important to know in advance which pathways could mediate resistance to the lapatinib treatment and to find ways of bypassing these obstacles [6].

Mesenchymal-epithelial transition factor protein (MET), the hepatocyte growth factor (HGF) receptor, is a 190-kDa RTK, and plays a critical role in tumor growth, invasion and metastasis. MET is frequently overexpressed and activated in a subset of GC [7]. Previously it has been shown that co-expression of MET and HER2 in GC is associated with poorer survival compared to overexpression of either one [8]. Moreover, MET overexpression occurred more frequently in HER2-positive GCs than in HER2-negative GCs [9]. Growing evidences implicate the interplays between HER family receptors and MET in cancer cells through overlapping downstream signaling pathways [6]. In vitro cell culture experiments showed that HGF-induced MET activation was responsible for lapatinib resistance in HER2-positive GC cell lines [10,11]. In addition, GC cells derived from HER2-positive and MET-positive GC showed that the combination of lapatinib and MET-inhibitor offered a more profound cell growth inhibition than lapatinib alone [9]. Despite the strong evidence regarding the interplay between MET and HER2 in GC, the current understanding of the regulation of MET expression and activation in relation to lapatinib-resistance in HER2-positive cells requires additional research.

Forkhead box O1 (FOXO1) is a transcription factor and a member of the FOXO subfamily of the Forkhead/winged helix family [10]. Since FOXO1 activates or represses multiple target genes, and consequently regulates a variety of cellular functions [11], dysregulation of FOXO1 would subsequently result in various disease states such as cancer. FOXO1 inactivation has been documented in several cancers, including GC [12], and its association with several anti-cancer drugs has increasing attracted oncologists' attention [13-15].

The existence of a negative crosstalk between FOXO1 and HER2 in parental GC cell lines was previously reported [16]. This crosstalk was associated with cancer cell growth, epithelial-mesenchymal transition, cell migration and invasion in vitro as well as tumorigenicity and metastasis in vivo [16]. However, the relationship between FOXO1 and anti-HER2 drug resistance in GC has not been reported. In the present study, lapatinib-resistant GC cell lines (SNU-216 LR 2-8) were generated by chronic exposure to lapatinib and the potential role of FOXO1 in lapatinib resistance was examined. In addition, we silenced MET expression and investigated its implication in the lapatinib resistance in the lapatinib-resistant, HER2-positive GC cells.

\section{Materials and Methods}

\section{Cell culture}

A HER2-positive GC cell line SNU-216 was purchased from the Korean Cell Line Bank (Seoul, Korea). Cells were maintained in RPMI 1640 medium (Life Technologies, Grand Island, NY) containing 10\% fetal bovine serum (FBS; BioWest, Kansas City, MO) in a humidified atmosphere containing $5 \% \mathrm{CO}_{2}$ at $37^{\circ} \mathrm{C}$.

\section{Reagents and antibodies}

Lapatinib was purchased from Cell Signaling Technology (Berverly, MA), and cisplatin (CDDP) was purchased from Sigma (St. Louis, MO). Antibodies against phospho-

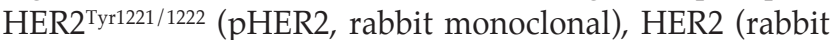
monoclonal), phospho-MET ${ }^{\mathrm{Tyr} 1234 / 1235}$ (pMET, rabbit monoclonal), phospho-AKT ${ }^{\text {Ser473 }}$ (pAKT, rabbit polyclonal), AKT (rabbit polyclonal), and FOXO1 (rabbit monoclonal) were purchased from Cell Signaling Technology. Antibodies against MET (rabbit polyclonal), $\beta$-actin (mouse monoclonal) and secondary antibodies, which are horseradish peroxidaseconjugated anti-rabbit IgG or anti-mouse IgG, were purchased from Santa Cruz Biotechnology (Santa Cruz, CA). 


\section{Generation of lapatinib-resistant clones SNU-216 LR from SNU-216 cells}

SNU-216 cells were cultured in the presence of increasing concentrations of lapatinib over a period of 8 months, reaching a final concentration of $10 \mu \mathrm{mol} / \mathrm{L}$ at the end of this period as described previously [17]. Single-cell clonal populations were obtained from a pool of resistant cells by serial dilutions. Cells were expanded in RPMI-1640 medium containing 10\% FBS and lapatinib $(1 \mu \mathrm{mol} / \mathrm{L})$.

\section{Growth inhibition assays}

The viability of cells was measured indirectly using crystal violet assay as described by Kim et al. [18]. Cells were seeded in 24-well plates at a density of $1 \times 10^{4}$ cells / well for cell growth and cultured for 4 days. To study the cytotoxicity effect of drugs, $1.5 \times 10^{4}$ cells were seeded in 24 -well plates, incubated for 24 hours, and treated for 3 days at $37^{\circ} \mathrm{C}$ with indicated concentrations of drugs dissolved in $0.04 \%$ dimethyl sulfoxide. Control columns contained cells without drug and blank columns contained medium alone. Medium was aspirated from the wells followed by washing three times with tap water. Attached cells were stained with $0.2 \%$ crystal violet aqueous solution in $20 \%$ methanol for 10 minutes followed by washing four times and were air dried. Crystal violet stain was dissolved in $10 \%$ sodium dodecyl sulfate (SDS) for 10 minutes at room temperature, transferred into 96-well plates, and the absorbance was measured at 570 nm using an enzyme-linked immunosorbent assay reader (Bio-Rad, Hercules, CA). The reading of blank columns was subtracted from each value.

\section{Western blot analysis}

Western blot analysis was performed as described previously [16]. Proteins were obtained when cells were subconfluent (70\%-80\%). Briefly, cell lysates in SDS lysis buffer (125 $\mathrm{mM}$ Tris- $\mathrm{HCl}$ [pH 6.8], 4\% SDS, $0.004 \%$ bromophenol blue, and $20 \%$ glycerol) were separated on 10\% SDS-polyacrylamide gel and electrophoretically transferred to polyvinylidene difluoride membranes (Millipore Corporation, Billerica, MA) blocked with 5\% non-fat dry milk in phosphatebuffered saline-Tween-20 (0.1\%, vol/vol) for 1 hour. The membranes were then incubated with a primary antibody against pHER2 (1:1,000), HER2 (1:1,000), pMET (1:1,000), MET $(1: 1,000), \operatorname{pAKT}(1: 1,000)$, AKT $(1: 1,000)$, FOXO1 $(1: 1,000)$, or $\beta$-actin $(1: 1,000)$. Horseradish peroxidase-conjugated anti-rabbit IgG $(1: 2,000)$ or anti-mouse $\operatorname{IgG}(1: 2,000)$ was used as a secondary antibody. Enhanced chemiluminescence (Amersham, Arlington Heights, IL) was used to detect the immunoreactive proteins. Equal protein loading was con- firmed by $\beta$-actin.

\section{Transfection of forkhead responsive element-luciferase construct and luciferase reporter assay}

To determine FOXO1 nuclear DNA-binding activity in GC cells, luciferase reporter assay was performed as previously described [15]. GC cells were seeded in 24-well plates at a density of $3 \times 10^{4}$ cells / well and were transiently cotransfected with $0.4 \mu \mathrm{g}$ forkhead responsive element (FHRE)luciferase reporter plasmid (reporter construct in which a small region of the Fas ligand promoter contains the three FHREs, Addgene plasmid 1789, Addgene Inc., Cambridge, MA) and $0.4 \mathrm{mg}$ pSV-b-galactosidase vector (Promega, Madison, WI), an internal control, using Lipofectamine Plus (Life Technologies). Twenty-four hours after transfection, assays for luciferase and $\beta$-galactosidase were carried out using a Dual-Luciferase Reporter Assay System (Promega). Luciferase activity was measured on an Auto-Lumat LB 9505c luminometer (Berthold Analytical Instruments, Nashua, Germany) and was normalised by $\beta$-galactosidase activity.

\section{Overexpressing FOXO1 in SNU-216 LR cells}

Overexpressing FOXO1 was done by stable transfection of pcDNA3 containing human FOXO1A3 mutant gene (Addgene plasmid 13508, Addgene Inc.). The plasmid FOXO1A3 encodes a constitutively active version of FOXO1 (all three AKT phosphorylation sites are mutated to Ala). Each vector $(1 \mu \mathrm{g})$ was transfected into GC cells using Lipofectamine Plus according to the manufacturer's instructions. Twenty-four hours after transfection, G418 $(3 \mu \mathrm{g} / \mathrm{mL})$ was added to select stable FOXO1A3 clones.

\section{Lentivirus-mediated short hairpin RNA silencing of HER2}

Lentiviral particles containing non-targeting short hairpin RNA (shRNA) or HER2 shRNA were purchased (Sigma). The sequence of HER2 shRNA was 5'-CCGGTGTCAGTATCCA GGCTTTGTACTCGAGTACAAAG CCTGGATACTGACATTTTTG-3'. The control shRNA particles contain 4 bp mismatches within the short hairpin sequence to any known human or mouse gene. Viral infection was performed by incubating GC cells in the culture medium containing lentiviral particles for 12 hours in the presence of $5 \mu \mathrm{g} / \mathrm{mL}$ Polybrene (Santa Cruz Biotechnology). Pooled puromycin $(2 \mu \mathrm{g} / \mathrm{mL})$-resistant cells were used for further analysis. 
A

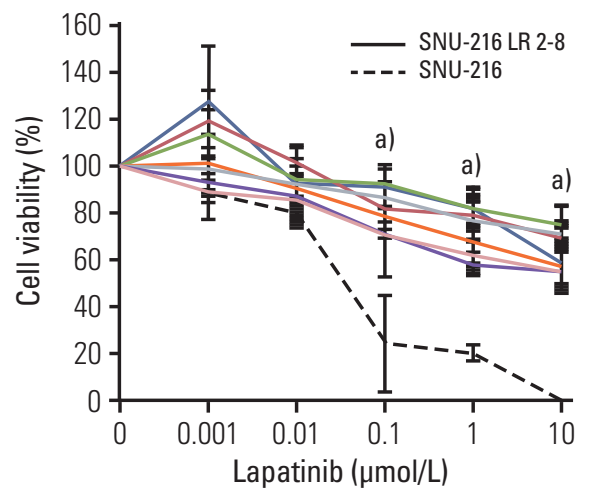

SNU-216 LR
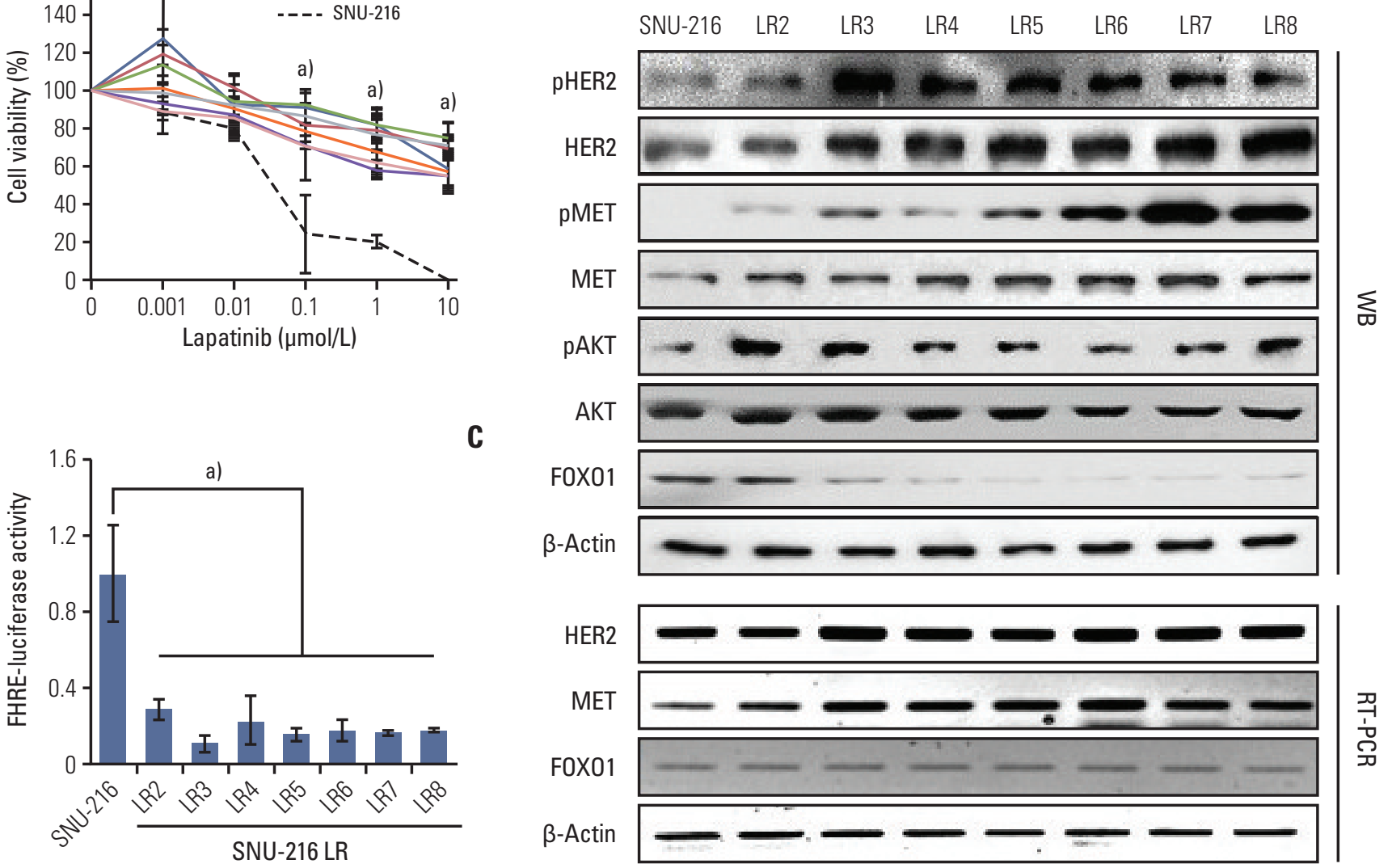

Fig. 1. Effect of chronic lapatinib treatment on SNU-216 cells. (A) Lapatinib-resistant (LR), human epidermal growth factor receptor 2 (HER2)-positive gastric cancer (GC) cell lines (SNU-216 LR2-8) were generated from a lapatinib-sensitive, HER2positive SNU-216 GC cell line by chronic exposure to lapatinib over a period of 8 months. Twenty-four hours after plating, parental and lapatinib-resistant (LR) SNU-216 cells were treated with the indicated concentrations of lapatinib for 3 days, and cell viability was determined using crystal violet assay. The percentage of viable cells is shown relative to untreated cells (considered as 100\%). (B) Comparative analysis of total and phosphorylated HER2, MET, and AKT as well as total forkhead box O1 (FOXO1) by Western blot analysis (WB). mRNA expressions of HER2, MET, and FOXO1 were determined by reverse transcription-polymerase chain reaction (RT-PCR). $\beta$-Actin protein and mRNA were served as loading controls. (C) FOXO1 transcriptional activity was determined by the luciferase reporter assay and was normalized by $\beta$-galactosidase activity. Luciferase activity in parental SNU-216 cells was arbitrarily set to 1 . Each bar represents the mean \pm standard deviation. ${ }^{\text {a) }} \mathrm{p}<0.05$ vs. parental SNU-216 cells.

\section{MET silencing by stable transfection with shRNA plas- mid vector}

For MET silencing, the pGFP-V-RS plasmid vectors containing either scrambled shRNA or MET shRNA were purchased from OriGene (Rockville, MD). The sequence of MET shRNA was 5'-GCAAGCCAGATTCTGCCGAACCAATGGAT-3'. Each vector $(1 \mu \mathrm{g})$ was transfected into GC cells using Lipofectamine Plus according to the manufacturer's instructions. Twenty-four hours after transfection, puromycin $(2 \mu \mathrm{g} / \mathrm{mL})$ was added to select stable MET shRNA clones.

\section{Reverse transcription-polymerase chain reaction}

Reverse transcription-polymerase chain reaction (RT-PCR) was performed to determine the mRNA level of molecules in GC cells, and the amplification of $\beta$-actin transcripts was 
A

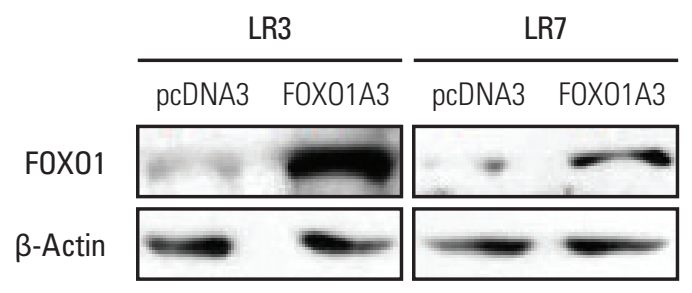

LR3

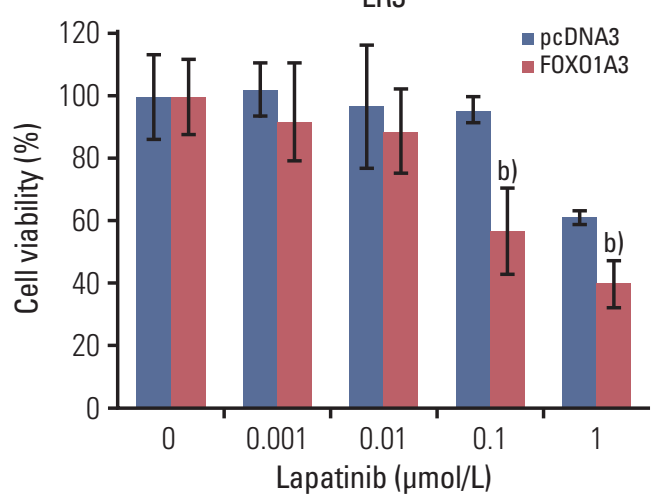

LR3

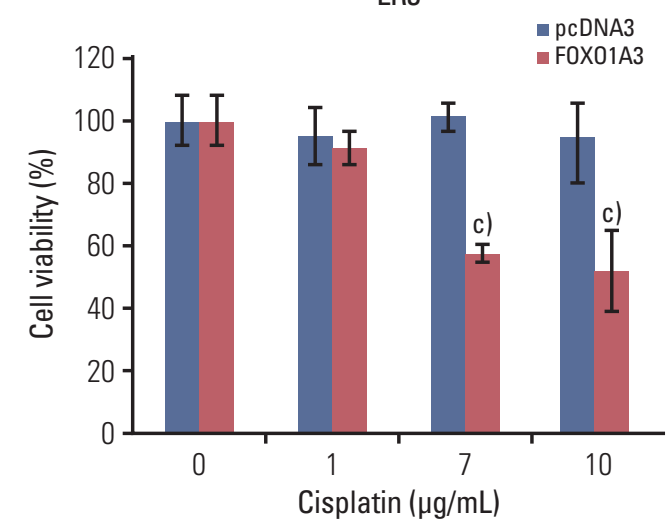

LR3

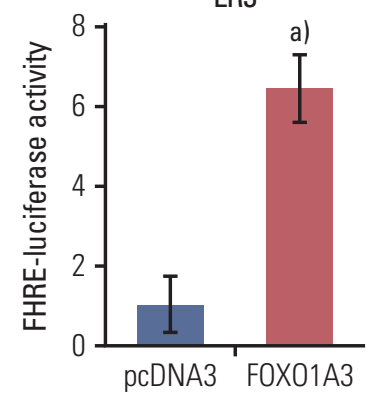

C

LR7

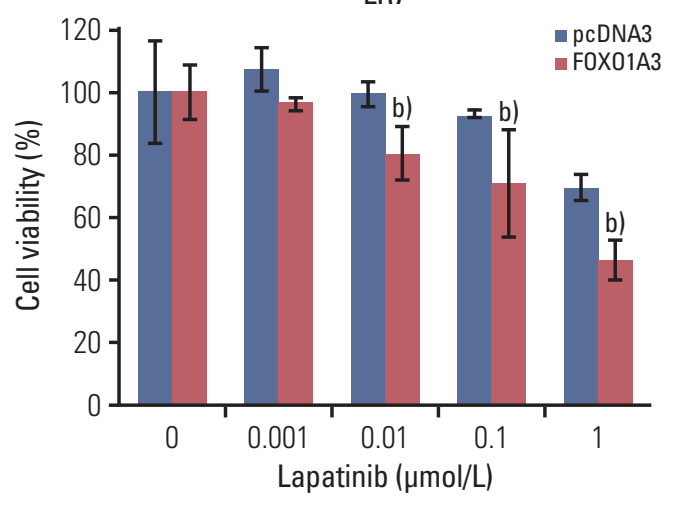

D

LR7

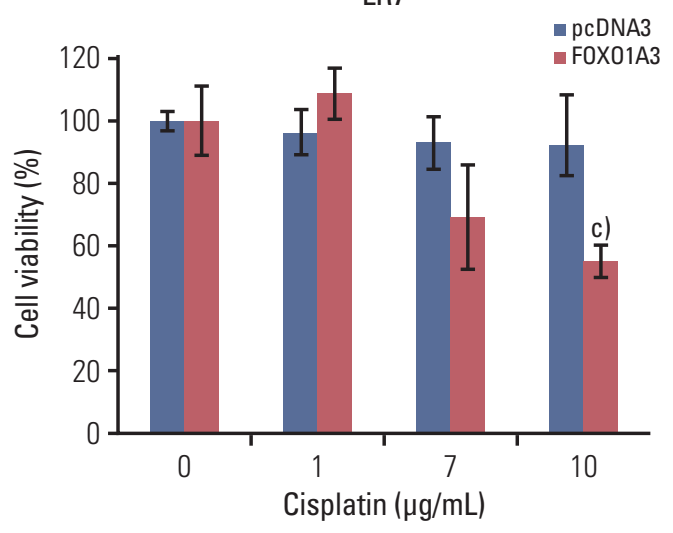

Fig. 2. Effect of forkhead box O1 (FOXO1) overexpression on lapatinib and/or cisplatin sensitivity in lapatinib-resistant (LR) cell lines. SNU-216 LR3 and LR7 cells were transfected with empty pcDNA3 vector (pcDNA3) or FOXO1A3 mutant vector (FOXO1A3). Cell viability was measured by crystal violet assay. (A) FOXO1 overexpression was confirmed by Western blot analysis. (B) FOXO1 transcriptional activity was analyzed by the luciferase reporter assay. (C) Cells were treated with the indicated concentrations of lapatinib, and cell viability was measured after 3 days. The percentage of viable cells is shown relative to untreated cells (considered as 100\%). (D) Cells were treated with the indicated concentrations of cisplatin, and cell viability was measured after 3 days. The percentage of viable cells is shown relative to untreated cells (considered as $100 \%$ ). (Continued to the next page) 

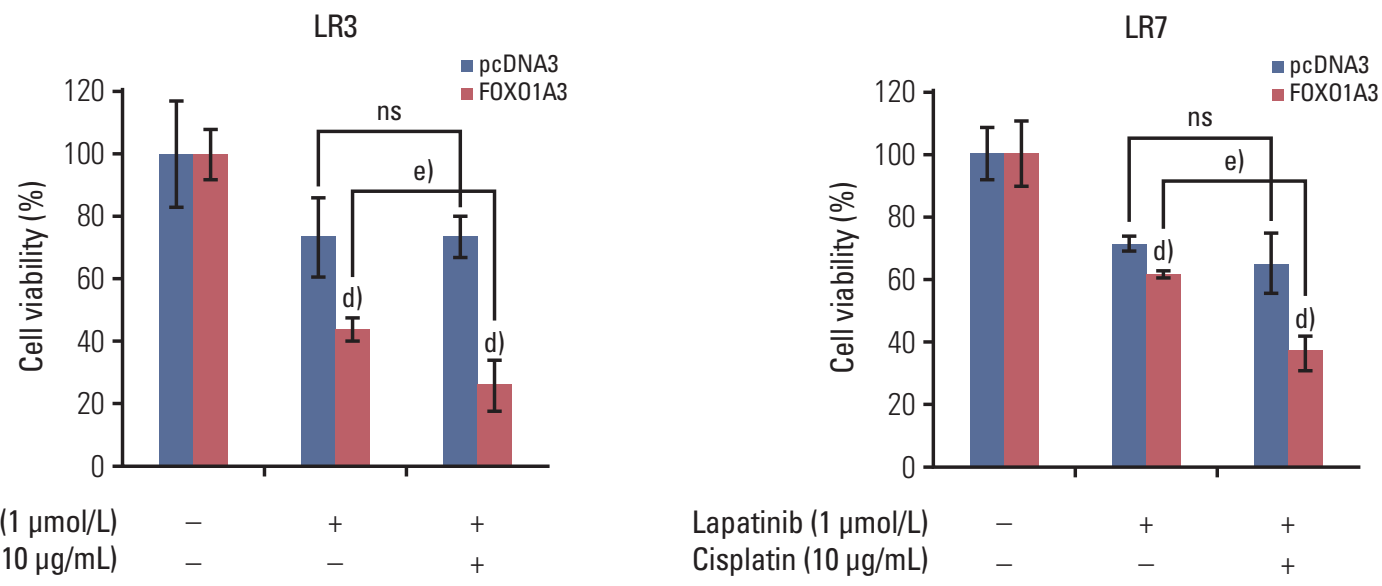

Fig. 2. (Continued from the previous page) (E) Cells were treated with the $1 \mu \mathrm{mol} / \mathrm{L}$ lapatinib alone or combined with $10 \mu \mathrm{g} / \mathrm{mL}$ cisplatin (CDDP), and cell viability was measured after 3 days. The percentage of viable cells is shown relative to untreated cells (considered as $100 \%$ ). Each bar represents the mean \pm standard deviation. ns, not significant. ${ }^{\text {a) }} p<0.05$ vs. pcDNA3 cells, ${ }^{b)} p<0.05$ vs. lapatinib-treated pcDNA3 cells, ${ }^{c} p<0.05$ vs. cisplatin-treated pcDNA3 cells, ${ }^{d} p<0.05$ vs. pcDNA3 cells, ${ }^{\text {e) }} \mathrm{p}<0.05$ vs. lapatinib-treated FOXO1A3 cells.

used as the control to normalize the transcript levels of molecules. Total RNAs were isolated using TRIZOL reagent (Invitrogen, Carlsbad, CA), and reverse-transcription was performed to synthesize cDNAs in a $20 \mu \mathrm{L}$ reaction mixture containing each gene-specific primer, $1 \mu \mathrm{g}$ of RNA, $2 \times$ reaction buffer, $0.4 \mu \mathrm{L}$ Taq polymerase, and $1.2 \mathrm{mM} \mathrm{MgCl}_{2}$. The cDNAs of HER2 transcripts were amplified for 28 cycles ( 30 seconds at $94^{\circ} \mathrm{C}, 30$ seconds at $52^{\circ} \mathrm{C}$, and 30 seconds at $70^{\circ} \mathrm{C}$ ), the cDNAs of MET transcripts were amplified for 30 cycles (30 seconds at $94^{\circ} \mathrm{C}, 30$ seconds at $52^{\circ} \mathrm{C}$, and 30 seconds at $72^{\circ} \mathrm{C}$ ), the cDNAs of FOXO1 transcripts were amplified for 25 cycles ( 30 seconds at $94^{\circ} \mathrm{C}, 1$ minute at $57^{\circ} \mathrm{C}$, and 1 minute at $72^{\circ} \mathrm{C}$ ), and the cDNAs of $\beta$-actin transcripts were amplified for 18 cycles $\left(94^{\circ} \mathrm{C}\right.$ for 30 seconds, $52^{\circ} \mathrm{C}$ for 30 seconds, and $70^{\circ} \mathrm{C}$ for 30 seconds). The PCR cycling numbers had been optimized to avoid the amplification saturation. Five microliter RT-PCR product was separated on $1 \%$ agarose gels, which were subsequently stained with ethidium bromide. Primer sequences were $5^{\prime}$-GGGAGAG AGTTCTGAGGATT$3^{\prime}$ and $5^{\prime}$-CGTCCGTAGAAAGGTAGTTG-3' for HER2, 5' TTGC CAGAGACATGTATGATAAAG- $3^{\prime}$ and $5^{\prime}$-CCAGCATTTTAGCATTACTT-3' for MET, 5'-GCAGATCTACGAGTGGATGGTC- ${ }^{\prime}$ and $5^{\prime}$-AAACTGTGATCCAGGGCTG TC $-3^{\prime}$ for FOXO1, and 5'-ACACCTTCTACAATGAGCTG-3' and $5^{\prime}$-CATGATGGAGTTGAAGGT AG-3' for $\beta$-actin.

\section{Statistical analysis}

All experiments were performed using triplicate cultures, and the results were expressed as the mean \pm standard deviation. Statistical analyses were conducted using GraphPad Prism software for Windows 7 (ver. 4, GraphPad Software, San Diego, CA). Data were evaluated by two-tailed Student's $t$ test. Differences with a $\mathrm{p}$-value of $<0.05$ were considered statistically significant.

\section{Results}

\section{Lapatinib-resistant, HER2-positive GC cells exhibit down- regulation of FOXO1}

To verify if FOXO1 is involved in the acquired lapatinib resistance in HER2-positive GC cells, stable lapatinib-resistant GC cell lines SNU-216 LR (LR2-LR8) were generated from lapatinib-sensitive parental SNU-216 cells by exposure to continuous treatment with lapatinib $(0-10 \mu \mathrm{mol} / \mathrm{L})$ over a period of 8 months as described previously by Kim et al. [17]. While parental SNU-216 cells treated with $10 \mu \mathrm{mol} / \mathrm{L}$ lapatinib displayed an almost complete abrogation of growth, the resistant cell lines showed significantly lower cell viability reduction 
than parental cell line (Fig. 1A).

Western blot analysis (Fig. 1B) confirmed HER2 overexpression and low expression of FOXO1 in parental SNU-216 cells as previously reported [16]. In SNU-216 LR cells (except LR2) with the acquired lapatinib resistance, the expression and activation (manifested by phosphorylated forms) of HER2 and MET increased with a more distinctive upregulation of MET. Consistently, phosphorylated AKT (common downstream signaling protein of HER2 and MET), but not total AKT, increased in SNU-216 LR cells. In contrast, FOXO1 expression decreased in most of resistant cell lines (L3-L8). Downregulation of FOXO1 activation in all lapatinib-resistant cell lines was confirmed using the luciferase reporter assay (Fig. 1C). In addition, RT-PCR analysis showed that mRNA expressions of HER2 and MET were increased in SNU-216 LR cells. In contrast, FOXO1 mRNA expression was not changed (Fig. 1B).

\section{FOXO1 overexpression reduces resistance to lapatinib}

To examine whether FOXO1 is related to the acquired lapatinib resistance in SNU-216 LR cells (LR3 and LR7), FOXO1 expression was modulated by transfection with a construct expressing constitutively active FOXO1 (FOXO1A3). Cells transfected with empty pcDNA3 vector were generated as control. Western blot analysis (Fig. 2A) and the luciferase reporter assay (Fig. 2B) confirmed that FOXO1 expression and transcriptional activity were increased in FOXO1A3-transfected cells compared to vector control cells. The role of FOXO1 in the acquired lapatinib resistance in HER2-positive GC cells was examined by comparing cell growth of SNU-216 LR cells with or without FOXO1 overexpression using crystal violet assay. Treatment of SNU-216 LR3 cells (Fig. 2C) with lapatinib $(1 \mu \mathrm{mol} / \mathrm{L})$ for 72 hours decreased cell viability to $\sim 60 \%$ in vector cells and $\sim 40 \%$ in FOXO1A3-transfected cells compared to untreated cells. This result demonstrated a significant difference in the lapatinib cytotoxicity between vector control cells and FOXO1A3-transfected cells ( $p=0.0099)$. Similar results were shown in SNU-216 LR7 cells $(p=0.006)$ (Fig. 2C).

3. Constitutive FOXO1 activation increases the cytotoxic effect of combined treatment with lapatinib and cisplatin compared to lapatinib alone

In the preliminary study, we found that parental SNU-216 cells were cisplatin-sensitive, but SNU-216 LR cells were cis-

A
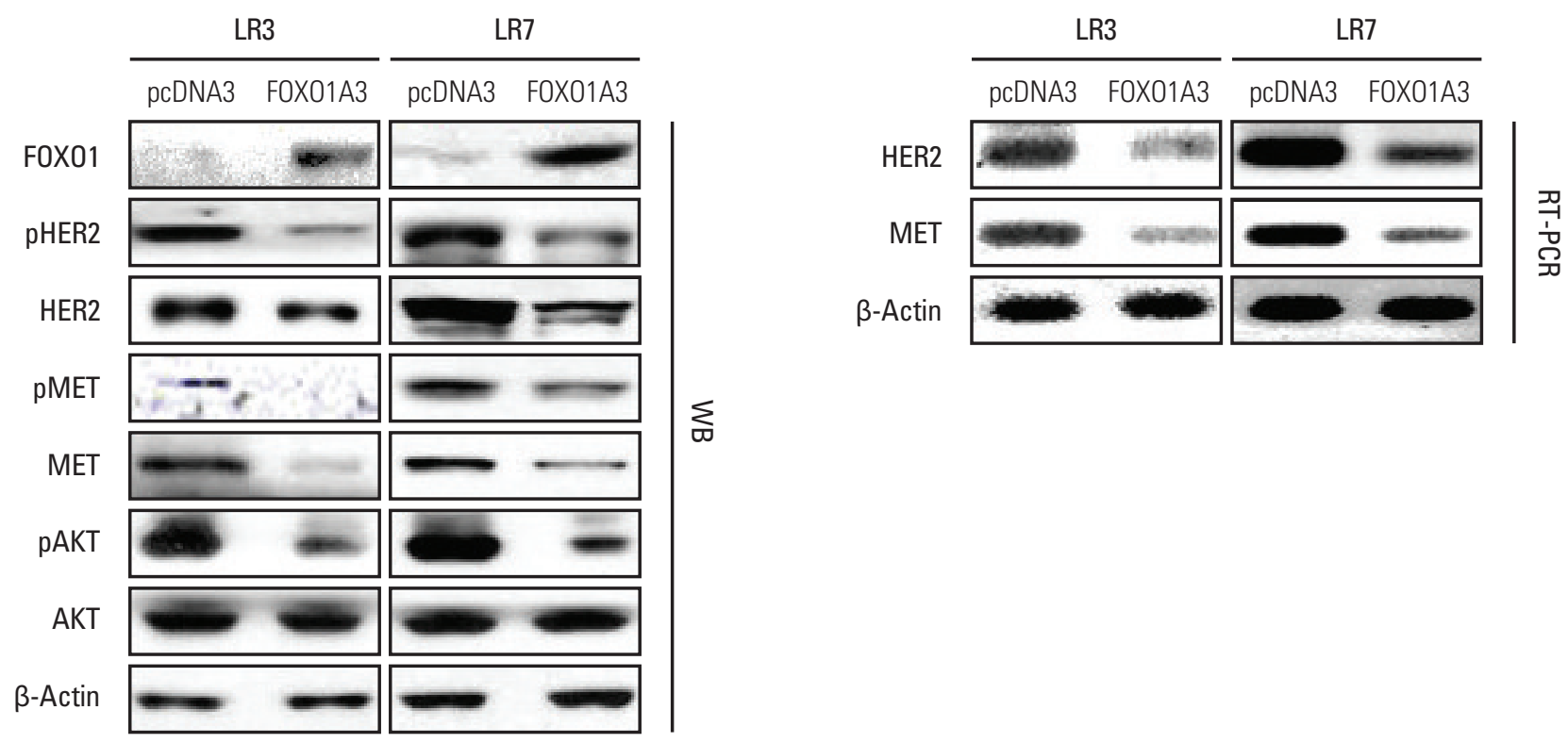

Fig. 3. Association between forkhead box O1 (FOXO1) and human epidermal growth factor receptor 2 (HER2)/MET in lapatinib-resistant (LR) cell lines. SNU-216 LR3 and LR7 cells were transfected with empty pcDNA3 vector (pcDNA3) or FOXO1A3 mutant vector (FOXO1A3). (A) The protein expressions of total and phosphorylated HER2, MET, and AKT were determined by Western blot analysis (WB). (B) The mRNA expressions of HER2 and MET were evaluated by reverse transcription-polymerase chain reaction (RT-PCR). 
A
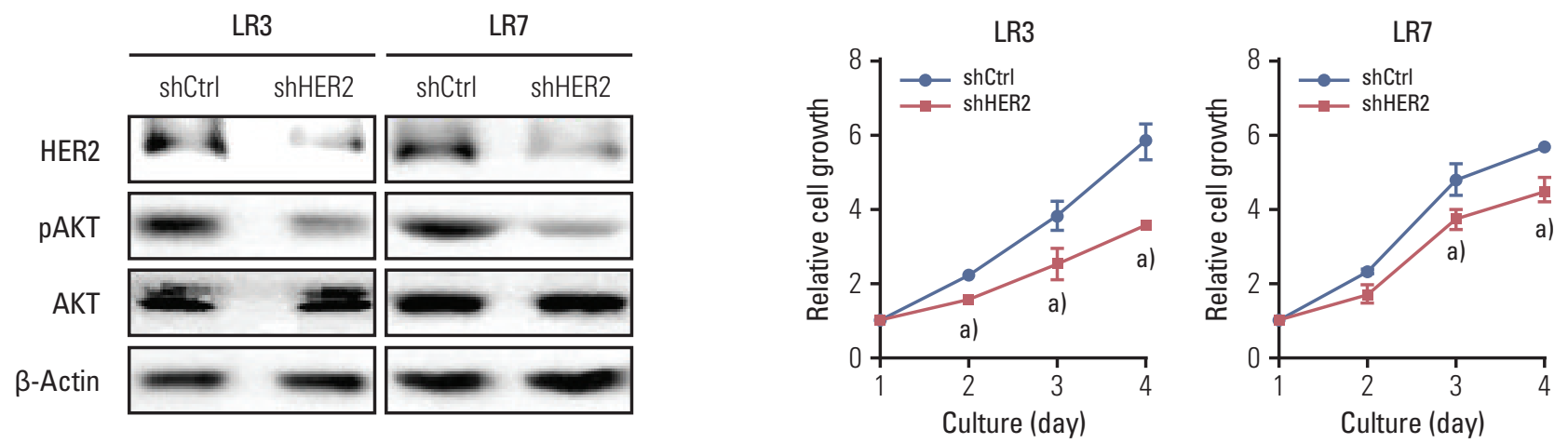

C
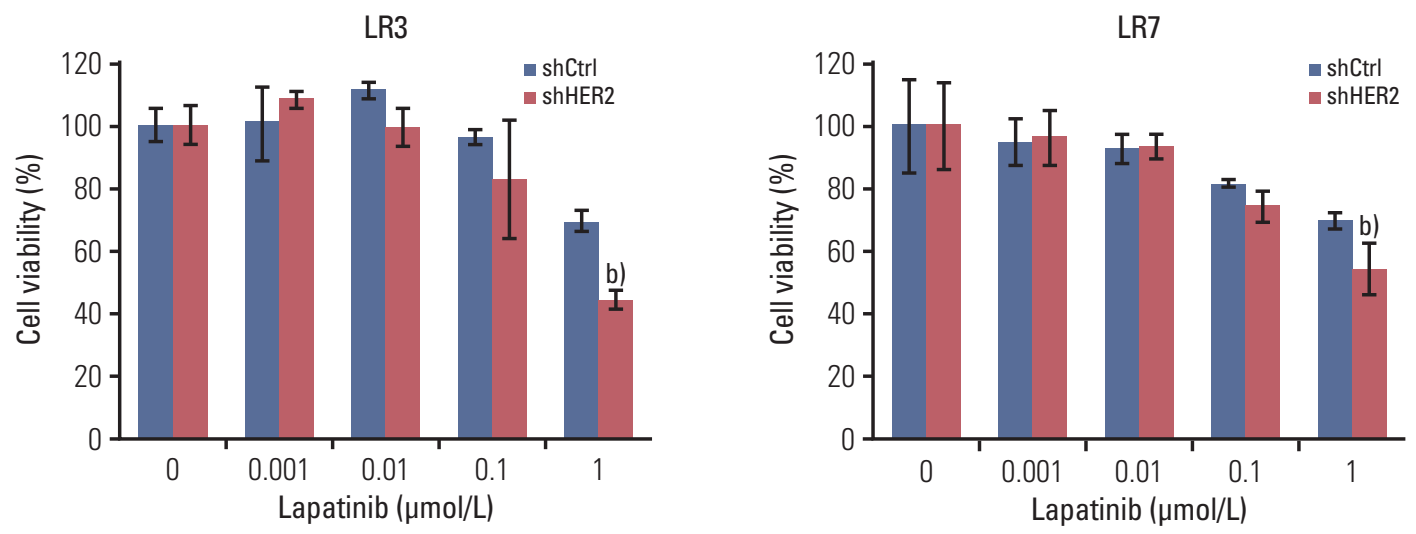

D
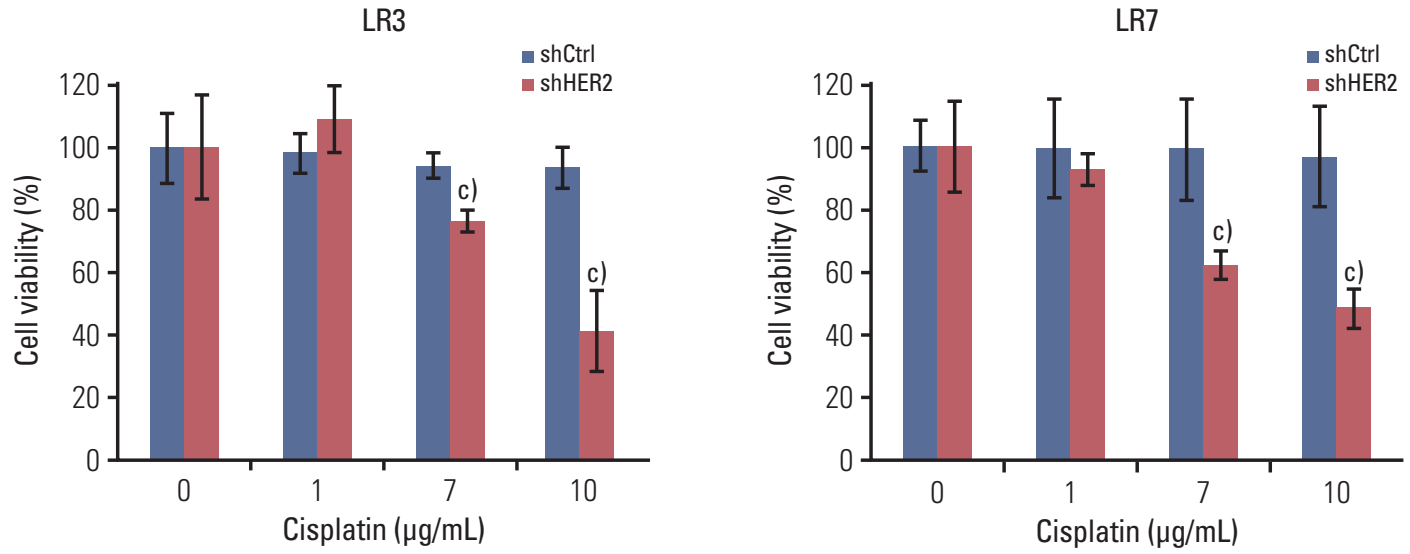

Fig. 4. Effect of human epidermal growth factor receptor 2 (HER2) downregulation on lapatinib/cisplatin resistance in lapatinib-resistant (LR) cell lines. SNU-216 LR3 and LR7 cells were infected with a lentivirus containing either control shRNA (shCtrl) or HER2 shRNA (shHER2). Cell viability was measured by crystal violet assay. (A) The protein expressions of HER2, pAKT, and AKT were determined by Western blot analysis. (B) Twenty-four hours after plating, cells were cultured for 3 days and cell growth was determined at the indicated times. (C) Cells were treated with the indicated concentrations of lapatinib, and cell viability was measured after 3 days. The percentage of viable cells is shown relative to untreated cells (considered as 100\%). (D) Cells were treated with the indicated concentrations of cisplatin, and cell viability was measured after 3 days. The percentage of viable cells is shown relative to untreated cells (considered as 100\%). (Continued to the next page) 
LR3

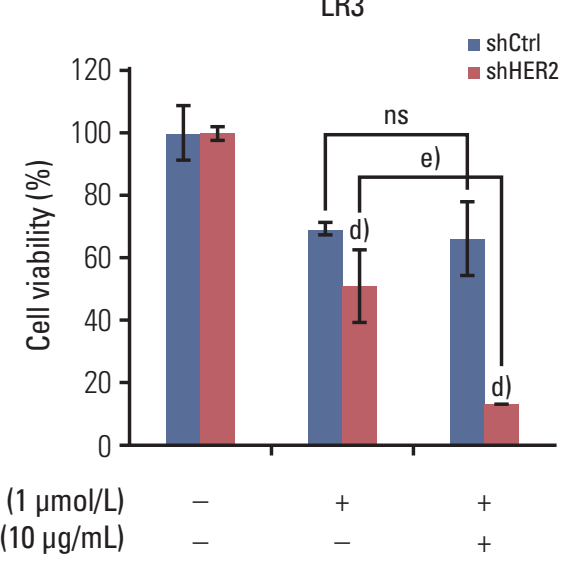

LR7

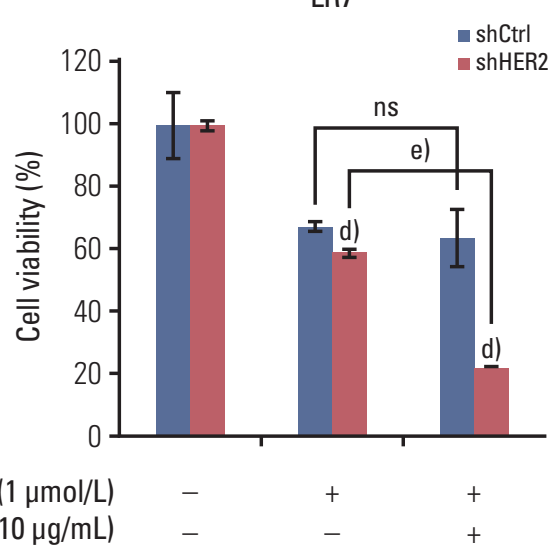

Fig. 4. (Continued from the previous page) (E) Cells were treated with the $1 \mu \mathrm{mol} / \mathrm{L}$ lapatinib alone or combined with $10 \mu \mathrm{g} / \mathrm{mL}$ cisplatin (CDDP), and cell viability was measured after 3 days. The percentage of viable cells is shown relative to untreated cells (considered as 100\%). Each bar represents the mean \pm standard deviation. ns, not significant. ${ }^{a} \mathrm{p}<0.05$ vs. shCtrl cells, ${ }^{b)} \mathrm{p}<0.05$ vs. lapatinib-treated shCtrl cells, ${ }^{\text {c) }} \mathrm{p}<0.05$ vs. cisplatin-treated shCtrl cells, ${ }^{\text {d) }} \mathrm{p}<0.05 \mathrm{vs}$. shCtrl cells, ${ }^{\text {e) }} \mathrm{p}<0.05$ vs. lapatinib-treated shHER2 cells.

platin-resistant (data not shown). Thus, SNU-216 LR cells with lapatinib resistance also developed cross-resistance to cisplatin. However, treatment of SNU-216 LR3 cells with cisplatin $(10 \mu \mathrm{g} / \mathrm{mL})$ in the presence of FOXO1 overexpression significantly decreased cell viability $(52 \%$ vs. the untreated control) compared with vector control cells ( $93 \%$ vs. the untreated control) ( $\mathrm{p}=0.016)$ (Fig. 2D). Similar results were shown in SNU-216 LR7 cells ( $\mathrm{p}=0.008$ ) (Fig. 2D).

Although lapatinib plus chemotherapy using parental SNU216 cells showed an additive or synergistic effect in vitro [19], inconsistent results were shown in the second-line treatment of patients with HER2-positive GC [20]. In the present study, the effect of adding cisplatin to lapatinib in SNU-216 LR3 and LR7 cells in the absence or presence of FOXO1 overexpression was examined. In the presence of FOXO1 overexpression, the combined treatment with cisplatin and lapatinib showed an additive cytotoxic effect ( $26 \%$ vs. the untreated control) in SNU-216 LR3 cells compared to treatment with lapatinib alone ( $44 \%$ vs. the untreated control) $(\mathrm{p}=0.022)$. However, there was no difference in cell viability in the absence of FOXO1 overexpression (Fig. 2E). Consistent results were shown in LR7 cells ( $\mathrm{p}=0.001$ ) (Fig. 2E). Thus, FOXO1 induces the efficacy of adding cisplatin to lapatinib in lapatinib-resistant SNU-216 LR cells.

\section{FOXO1 transcriptionally downregulates HER2 and MET}

HER2 and MET are strong predictors of lapatinib sensitivity in GC cells $[10,11,17]$. However, the molecular mechanism underlying a link between HER2 and MET with respect to lapatinib resistance remains undefined. To analyze the relationship between FOXO1 and HER2/MET in SNU-216 LR cells, we increased the FOXO1 expression and activation by transfection of FOXO1A3 as shown in Fig. 2A and B. In addition, Western blot analysis and RT-PCR were performed. Western blot analysis showed that the total and phosphorylated forms of HER2 and MET were downregulated by FOXO1 overexpression (Fig. 3A). Furthermore, the protein expression of phosphorylated AKT, but not the total AKT, was decreased. Consistently, RT-PCR demonstrated that mRNA expressions of HER2 and MET were downregulated (Fig. 3B). Taken together, FOXO1 negatively regulates HER2 and MET expressions at the transcriptional level, suggesting its involvement in the lapatinib-induced HER2 / MET signaling pathway.

\section{The acquired resistance to lapatinib and/or cisplatin is reversed by silencing HER2}

Parental SNU-216 cells are responsive to lapatinib because of the presence of HER2 overexpression. In the present study, however, SNU-216 cells became resistant to lapatinib after chronic exposure to lapatinib in spite of HER2 upregulation 
A

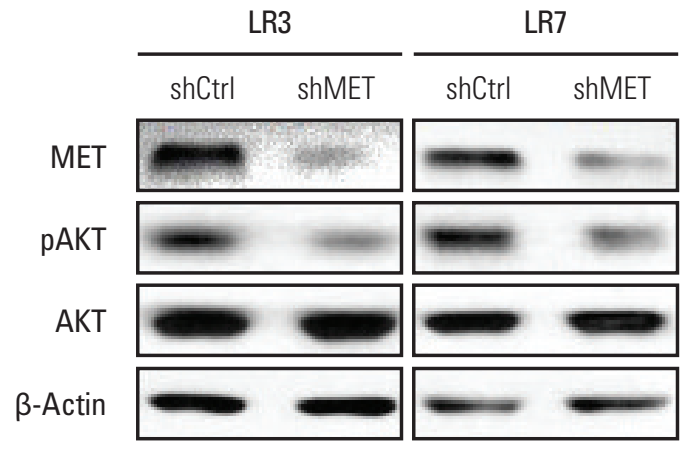

LR3

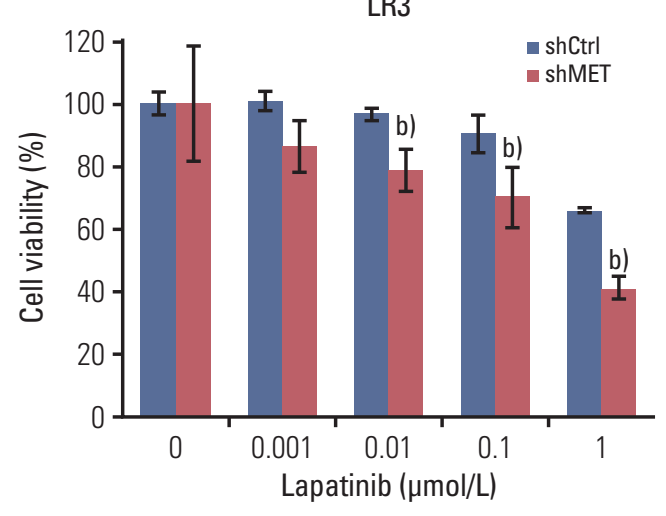

LR3

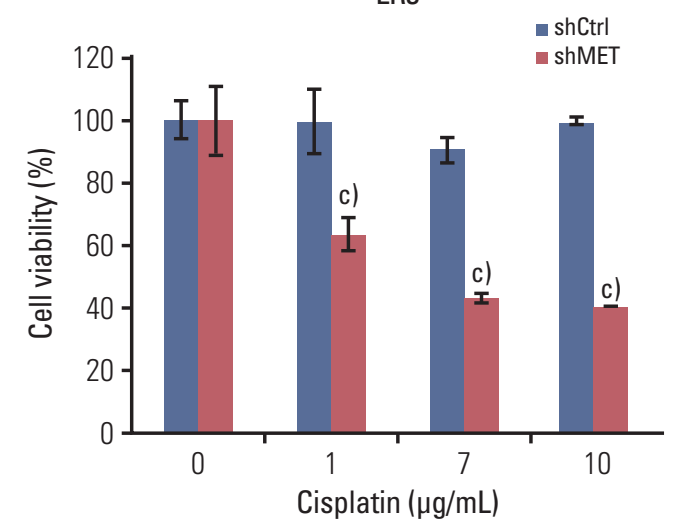

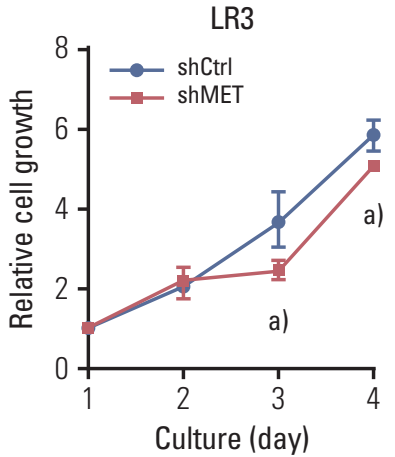

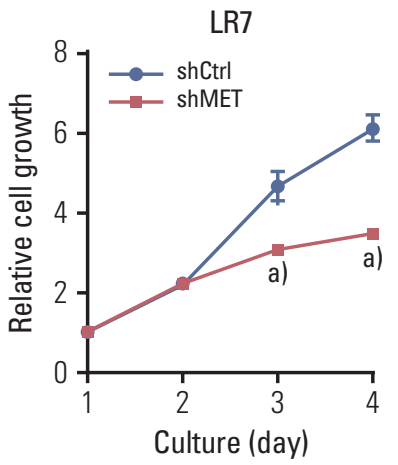

C

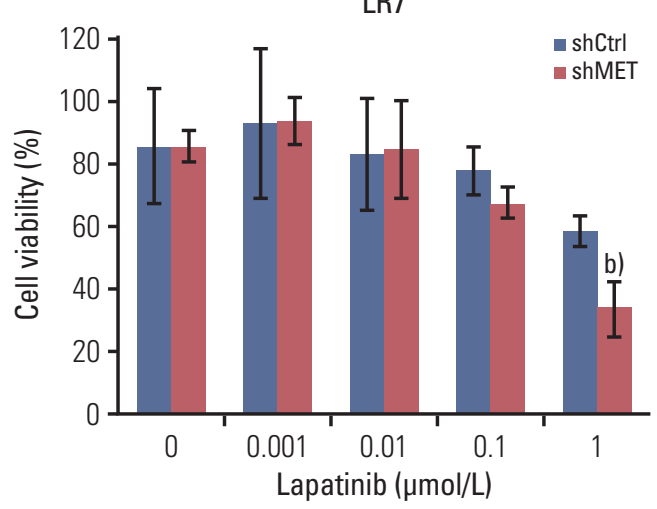

D

LR7

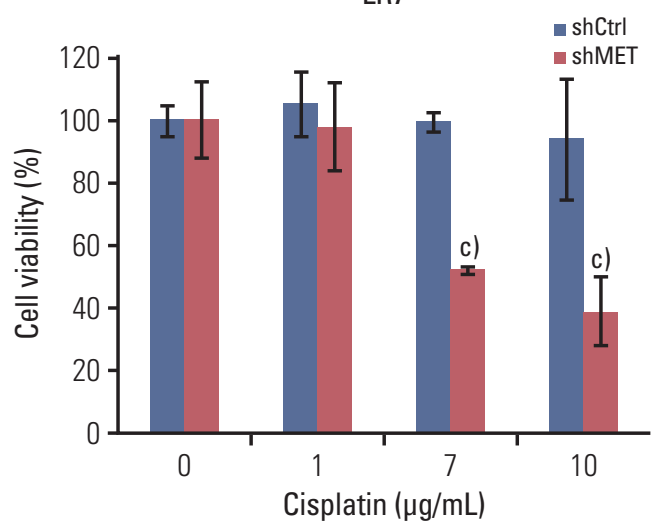

Fig. 5. Effect of MET downregulation on lapatinib / cisplatin resistance in lapatinib-resistant cell lines. SNU-216 LR3 and LR7 cells were transfected with pGFP-v-RS vectors containing either control scrambled shRNA (shCtrl) or MET shRNA (shMET). Cell viability was measured by crystal violet assay. (A) The protein expressions of MET, pAKT, and AKT were termined by Western blot analysis. (B) Cells were cultured for 3 days, and cell growth was determined at the indicated times. (C) Cells were treated with the indicated concentrations of lapatinib and cell viability was measured after 3 days. The percentage of viable cells is shown relative to untreated cells (considered as 100\%). (D) Cells were treated with the indicated concentrations of cisplatin, and cell viability was measured after 3 days. The percentage of viable cells is shown relative to untreated cells (considered as 100\%). (Continued to the next page) 
LR3

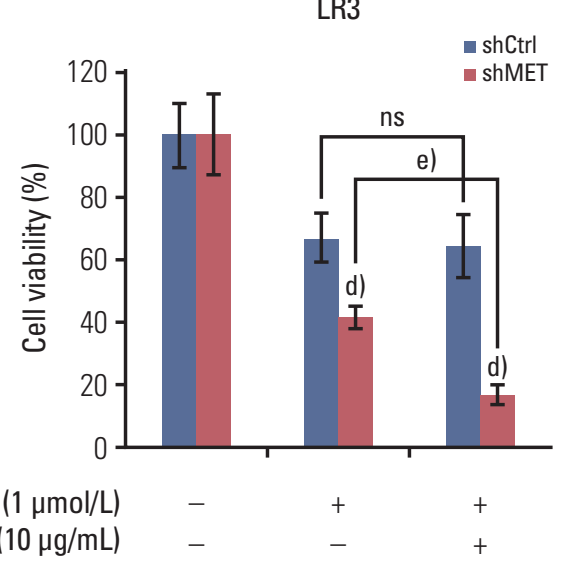

LR7

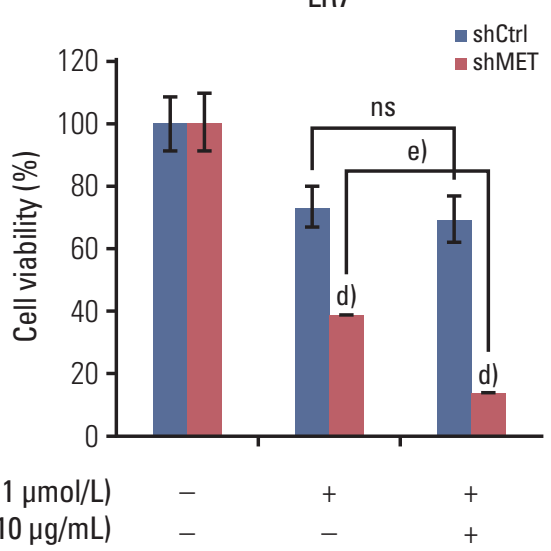

Fig. 5. (Continued from the previous page) (E) Cells were treated with the $1 \mu \mathrm{mol} / \mathrm{L}$ lapatinib alone or combined with $10 \mu \mathrm{g} / \mathrm{mL}$ cisplatin (CDDP), and cell viability was measured after 3 days. The percentage of viable cells is shown relative to untreated cells (considered as 100\%). Each bar represents the mean \pm standard deviation. ns, not significant. ${ }^{a} \mathrm{p}<0.05$ vs. shCtrl cells, ${ }^{b)} \mathrm{p}<0.05$ vs. lapatinib-treated shCtrl cells, ${ }^{\text {c) }} \mathrm{p}<0.05$ vs. cisplatin-treated shCtrl cells, ${ }^{\text {d) }} \mathrm{p}<0.05 \mathrm{vs}$. shCtrl cells, ${ }^{\text {e }} \mathrm{p}<0.05$ vs. lapatinib-treated shMET cells.

(Fig. 1A and B). To investigate whether the dependency of cell viability on HER2 expression persists in SNU-216 LR cells, HER2 was downregulated by RNAi (Fig. 4A). We found that HER2 shRNA-transfected cells had a lower level of pAKT (Fig. 4A) and showed growth inhibition (Fig. 4B). HER2 downregulation also significantly suppressed lapatinib resistance (Fig. 4C) and cisplatin resistance (Fig. 4D) compared to control shRNA cells. Furthermore, the combined treatment of HER2 shRNA-transfected SNU-216 LR cells showed an additive cytotoxic effect compared to treatment with lapatinib alone (Fig. 4E).

\section{The acquired resistance to lapatinib and/or cisplatin is reversed by silencing MET}

Parental SNU-216 cells showed a low level of MET expression, which notably increased in SNU-216 LR cells (Fig. 1B). To investigate the role of MET in lapatinib resistance in SNU216 LR cells, MET expression was downregulated by RNAi (Fig. 5A). MET shRNA-transfected cells had a lower level of pAKT (Fig. 5A) and also showed growth inhibition (Fig. 5B). Furthermore, MET silencing significantly suppressed lapatinib resistance (Fig. 5C) and cisplatin resistance (Fig. 5D) compared to control shRNA cells. In addition, combination treatment showed additional growth inhibition compared to treatment with lapatinib alone in MET shRNA-transfected cells, but not in control shRNA cells (Fig. 5E).

\section{HER2 and MET interplay through transcriptional control by FOXO1}

To investigate whether interplay between HER2 and MET exists, stable SNU-216 LR3 and LR7 cell lines overexpressing either HER2 shRNA (Fig. 6A) or MET shRNA (Fig. 6D) were used. Western blot analysis showed that HER2 silencing decreased the protein expressions of total and phosphorylated MET (Fig. 6A). In turn, MET silencing reduced the protein expressions of total and phosphorylated HER2 (Fig. 6D). Thus, these findings indicate that there is a positive interplay between these two molecules. Then, the effect of HER2 downregulation on FOXO1 expression and activation was examined. Western blot analysis (Fig. 6A) and luciferase reporter assay (Fig. 6B) demonstrated that HER2 silencing increased protein expression and activation of FOXO1 without a change in FOXO1 mRNA expression as shown by RT-PCR (Fig. 6C). Similar findings were observed in MET shRNA-transfected cells (Fig. 6D-F). These results indicate that FOXO1 expression is negatively regulated at the post-transcriptional level by HER2 and MET. 
A

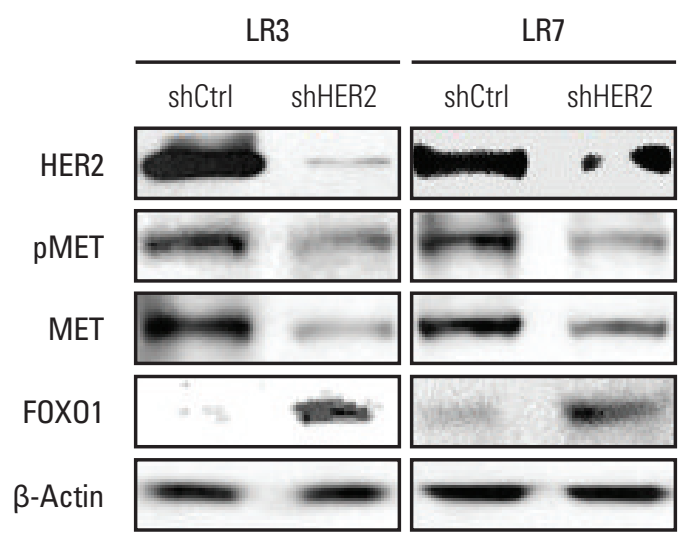

LR3

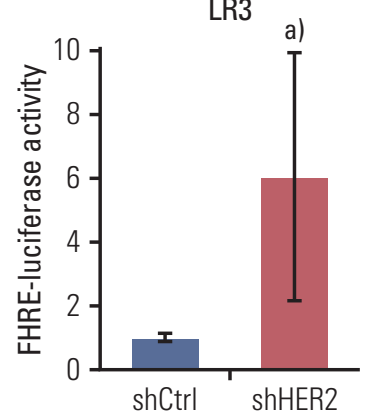

LR7

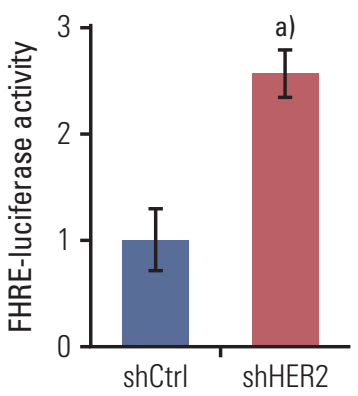

C
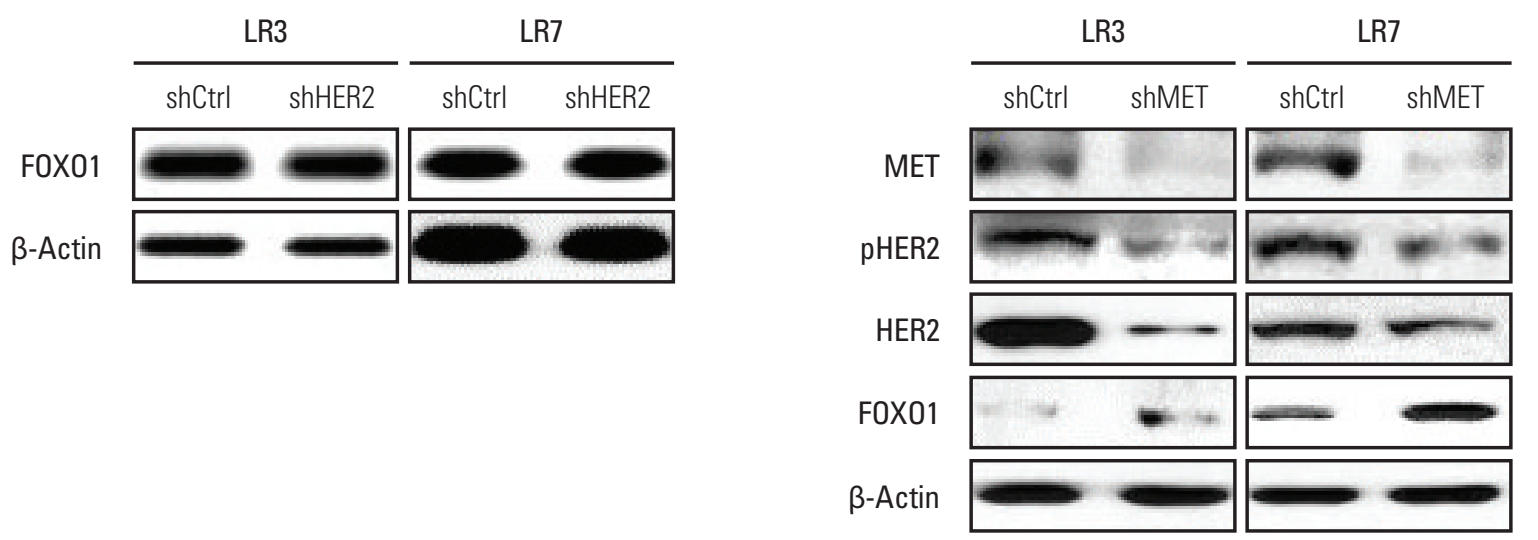

E

LR3

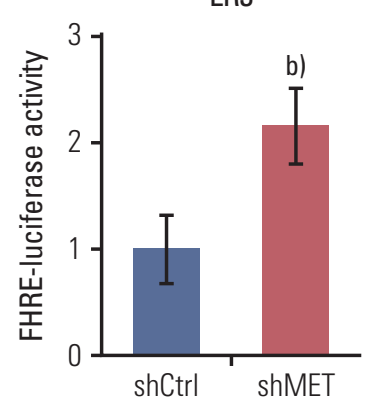

LR7

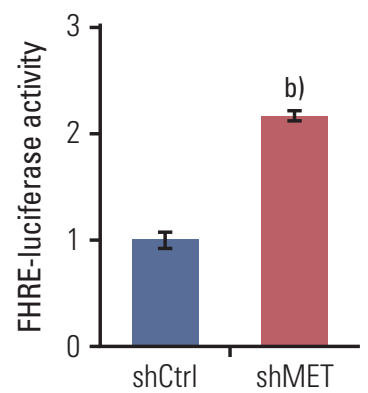

D 


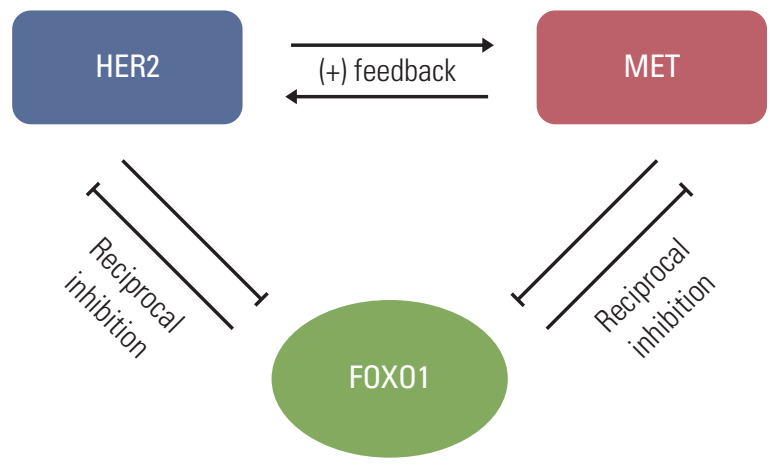

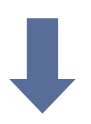

Sensitivity

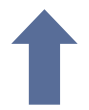

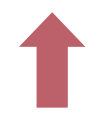

Resistance

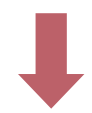

Fig. 7. Model for forkhead box O1 (FOXO1)-dependent acquired lapatinib resistance and the crosstalk among FOXO1, human epidermal growth factor receptor 2 (HER2) and MET in lapatinib-resistant, HER2-positive gastric cancer (GC) cells. Downregulation of FOXO1 leads to coactivation of HER2 and MET, which are essential to lapatinib resistance. Reintroduction of FOXO1 is necessary to reduce the lapatinib resistance in a subpopulation of HER2-positive GC patients showing lapatinib resistance.

\section{Discussion}

The acquisition of drug resistance in treated patients has become a significant issue in the establishment of strategy for human cancer therapy. Moreover, the complex interplay of signal-transduction pathways further complicates customizing of cancer treatments which might target a single mechanism [21]. The purpose of the present study was to determine the correlation between FOXO1 expression profile and the sensitivity to lapatinib alone or in combination with cisplatin, thereby providing a new strategy for treating lapatinib-resistant, HER2-positive GC. Here, FOXO1 suppression was identified as a determinant of acquired lapatinib resistance in HER2-positive GC cells, at least in part, through negative crosstalks with HER2 and MET. To the best of our knowledge, this is the first study to demonstrate the involvement of FOXO1 in anti-HER2 drug resistance and its association with MET in GC cells.

Given that HER2 serves as a putative target for therapy in HER2-positive GC, elucidating the molecular mechanism of lapatinib resistance is critical to establish a more efficient treatment strategy for patients who failed to respond to adjuvant trastuzumab-based chemotherapy. However, the molecular mechanism underlying the unresponsiveness of GC to lapatinib remains largely unexplained. Previously, lapatinib sensitivity was shown to be positively correlated to the degree of HER2 overexpression in various cancer cells [10]. Consistently, lapatinib responsiveness was shown in HER2-positive parental GC cell lines SNU-216 and NCI-N87 [19]. Although the initially addicting oncoprotein HER2 in parental SNU-216 cells is the target of lapatinib, SNU-216 LR cells were lapatinib-resistant in spite of HER2 upregulation.
This suggests that the acquired lapatinib resistance in SNU216 LR cells may be attributed to an alternative or redundant survival pathway [22]. Indeed, Kim et al. [17] suggested that MET upregulation could confer the acquired lapatinib resistance to lapatinib-sensitive, HER2-positive GC cells.

Previous studies have shown that FOXO1 plays an important role in the regulation of responsiveness of cancer cells to various anticancer drugs [13-15]. For example, FOXO1 increased paclitaxel resistance in ovarian cancer cells [13], adriamycin resistance in breast cancer cells [14] and cisplatin resistance in GC cells [15]. With respect to HER2-positive cancer cells, FOXO1 decreased trastuzumab resistance in HER2-positive breast cancer cells [23,24]. However, different resistance mechanisms have been reported for trastuzumab and lapatinib [25], and lapatinib sensitivity in cultured cells is determined by tissue type [26]. In the present study, FOXO1 activation was downregulated in SNU-216 LR cells compared to parental SNU-216 cells. Thus, the present study hypothesized that FOXO1 is implicated in the acquired lapatinib resistance in these cells in association with HER2 and MET. Taking advantage of lapatinib-resistant, HER2-posistive SNU-216 LR cell lines, we confirmed that lapatinib alone showed weak growth inhibitory effect toward SNU-216 LR cells. Further, FOXO1 overexpression in these cells induced an enhanced cytotoxic effect of lapatinib. These results demonstrate the importance of FOXO1 for the lapatinibmediated cytotoxic effect in SNU-216 LR cells.

The efficacy of lapatinib alone or in combination with standard chemotherapy for HER2-positive GC is yet to be improved. In the preclinical cell-based study using HER2positive GC cell lines (SNU-216 and NCI-N87), lapatinib plus chemotherapy showed an additive or synergistic effect [19]. In contrast, a randomized, open-labeled, phase III study 
(Tytan study) showed that second-line treatment of HER2positive advanced GC patients with lapatinib plus chemotherapy did not significantly improve overall survival compared to chemotherapy alone [20]. In the present study, the combined treatment with lapatinib and cisplatin did not induce a significant difference in cell viability of SNU-216 LR cells compared to lapatinib treatment, which is consistent with results of the clinical trial. In the presence of FOXO1 overexpression, however, combined treatment resulted in a greater reduction in cell viability compared to treatment with lapatinib alone. These results provides a direct evidence that FOXO1 suppression confers acquired resistance to lapatinib and / or cisplatin in lapatinib-resistant, HER2-positive GC cells. Since FOXO1 involvement in lapatinib resistance has not been previously identified in HER2-positive cancers, this is a novel molecular mechanism underlying acquired lapatinib resistance in HER2-positive GC. These findings provide a basis for the proposal that systemic reintroduction of FOXO1 in HER2-positive GC patients could result in a selective lapatinib toxicity in cancer cells. In the near future, it should be possible to generate an enhanced anti-cancer effect via a combination of lapatinib and FOXO1-replacement therapy. However, the technique of transcription factor-replacement therapy is not yet in general use.

The phenomenon of oncogene addiction has revealed potentially important therapeutic opportunities that can lead to the selective elimination of tumor cells showing dependence on a protein or pathway [22]. Just as acute inactivation of addicting oncoproteins frequently leads to cancer cell death, recent evidence points to similar outcomes induced by the reintroduction of a wild-type version of tumor suppressor genes that are frequently inactivated in cancer cells [22]. Accumulating data on the "addiction to lack of tumor suppressor genes" indicate that, in the establishment of the oncogene addicted state, a prerequisite may involve the removal of support systems such as tumor suppressors (p53, FHIT, PTEN, LKB1, and TESTIN) [22]. In the present study using SNU-216 LR cells, upregulations of oncogenes HER2 and MET as well as downregulation of tumor suppressor FOXO1 were observed. In addition, FOXO1 overexpression reduced mRNA expressions of HER2 and MET. Accordingly, it seems that chronic exposure to lapatinib induced FOXO1 downregulation, and consequently upregulated of HER2 and MET through transcriptional control. Taken together, we speculate that FOXO1 inactivation may be a prerequisite in the establishment of the addiction to HER2 as well as MET in SNU-216 LR cells.

Although lapatinib responsiveness is associated with HER2 overexpression in parental GC cell lines [19], HER2 was upregulated in lapatinib-resistant, HER2-positive GC cell lines in the present study. However, the effect of HER2 modulation on the acquired lapatinib resistance in these cells has never been evaluated. This study investigated whether the loss of HER2 addiction or addiction switching to an alternative oncogene [22] is induced by chronic exposure to lapatinib. HER2 expression in SNU-216 LR cells was downregulated by using RNA interference, which resulted in AKT inactivation and cell growth suppression. These results indicate that HER2 addiction still remains. Inconsistently, HER2 downregulation significantly suppressed resistance to an anti-HER2 drug lapatinib, In addition, cisplatin resistance was decreased in HER2-silenced SNU-216 LR cells, which is consistent with a previous report [27].

MET has been shown to cross-react with EGFR proteins and possibly substitutes for their activity, thus conferring resistance to EGFR-targeting drugs [28]. With respect to GC cells, HGF-induced MET activation in HER2-positive GC cell lines (SNU-216 and NCI-N87) induced lapatinib resistance [10]. In the present and previous [17] studies, chronic exposure to lapatinib upregulated MET compared to parental HER2-positive GC cells. However, the effect of MET modulation on the acquired lapatinib resistance in these cells has not been shown. This study examined whether cancer cells exhibit MET addiction in addition to original HER2 addiction. MET downregulation in SNU-216 LR cells decreased AKT activation and cell growth, which was similar to results obtained with HER2 downregulation. Consistently, MET downregulation significantly suppressed lapatinib and/or cisplatin resistance. Since both HER2 and MET contributed to lapatinib resistance in SNU-216 LR cells, it seems that MET provides redundant survival signals through the activation of downstream survival pathways that overlap with those of HER2. Thus, SNU-216 LR cells can be considered to be "co-addicted" to HER2 and MET.

The present study showed that MET expression and activation were clearly lower than those of HER2 in parental SNU-216 cells. After acquisition of lapatinib resistance, MET was notably upregulated in the majority of SNU-216 LR cell lines compared to HER2. Previously, MET amplification was shown to be responsible for the MET overexpression induced by EGFR RTK inhibitor treatment of non-small cell lung cancer patients who displayed acquired resistance [22]. However, Kim et al. [17] reported that there is no MET gene amplification in HER2-positive SNU-216 LR cells with acquired lapatinib resistance. Although concomitant overexpression of HER2 and MET was observed in a subset of GC patients [9], the regulatory relationship between these two molecules has not been demonstrated in GC. Our results showed that HER2 downregulation in SNU-216 LR cells suppressed the expressions of both total and phosphorylated MET and vice versa. Thus, it seems that the interplay between these two molecules in the survival signaling pathway is not due to transphosphorylation, instead is due to the activations of overlapping downstream molecules. Since 
FOXO1 is negatively controlled by and controls HER2 and MET, FOXO1 seems to serve as an important linker between HER2 and MET signaling pathways via negative crosstalks. In addition, downregulation of either HER2 or MET increased FOXO1 protein expression and activation, but not mRNA expression, which indicates negative regulation at the post-transcriptional level.

In conclusion, the present study shows a novel molecular mechanism that could cause the acquired lapatinib resistance in a subset of HER2-positive GC cells. It seems that FOXO1 suppression is implicated in the acquisition of lapatinib resistance in HER2-positive GC cells through upregulation of MET as well as HER2 (Fig. 7). Thus, this should be taken into consideration when designing combination therapies for a subset of lapatinib-resistant, HER2-positive GC.

\section{Conflicts of Interest}

Conflict of interest relevant to this article was not reported.

\section{Acknowledgments}

This work was supported by grant no 04-2015-0290 from the SNUH Research Fund and the Education and Research Encouragement Fund of Seoul National University Hospital (2017).

\section{References}

1. Gravalos C, Jimeno A. HER2 in gastric cancer: a new prognostic factor and a novel therapeutic target. Ann Oncol. 2008;19: 1523-9.

2. Bang YJ, Van Cutsem E, Feyereislova A, Chung HC, Shen L, Sawaki A, et al. Trastuzumab in combination with chemotherapy versus chemotherapy alone for treatment of HER2-positive advanced gastric or gastro-oesophageal junction cancer (ToGA): a phase 3, open-label, randomised controlled trial. Lancet. 2010;376:687-97.

3. Liu L, Wu N, Li J. Novel targeted agents for gastric cancer. J Hematol Oncol. 2012;5:31.

4. Spector NL, Xia W, Burris H 3rd, Hurwitz H, Dees EC, Dowlati A, et al. Study of the biologic effects of lapatinib, a reversible inhibitor of ErbB1 and ErbB2 tyrosine kinases, on tumor growth and survival pathways in patients with advanced malignancies. J Clin Oncol. 2005;23:2502-12.

5. Engelman JA, Zejnullahu K, Mitsudomi T, Song Y, Hyland C, Park JO, et al. MET amplification leads to gefitinib resistance in lung cancer by activating ERBB3 signaling. Science. 2007; 316:1039-43.

6. Corso S, Ghiso E, Cepero V, Sierra JR, Migliore C, Bertotti A, et al. Activation of HER family members in gastric carcinoma cells mediates resistance to MET inhibition. Mol Cancer. 2010; 9:121

7. Lee HE, Kim MA, Lee HS, Jung EJ, Yang HK, Lee BL, et al. MET in gastric carcinomas: comparison between protein expression and gene copy number and impact on clinical outcome. Br J Cancer. 2012;107:325-33.

8. Nakajima M, Sawada H, Yamada Y, Watanabe A, Tatsumi M, Yamashita J, et al. The prognostic significance of amplification and overexpression of c-met and c-erb B-2 in human gastric carcinomas. Cancer. 1999;85:1894-902.

9. Ha SY, Lee J, Jang J, Hong JY, Do IG, Park SH, et al. HER2-positive gastric cancer with concomitant MET and/ or EGFR overexpression: a distinct subset of patients for dual inhibition therapy. Int J Cancer. 2015;136:1629-35.

10. Chen CT, Kim H, Liska D, Gao S, Christensen JG, Weiser MR. MET activation mediates resistance to lapatinib inhibition of HER2-amplified gastric cancer cells. Mol Cancer Ther. 2012;11:660-9.

11. Zhang Z, Wang J, Ji D, Wang C, Liu R, Wu Z, et al. Functional genetic approach identifies MET, HER3, IGF1R, INSR pathways as determinants of lapatinib unresponsiveness in HER2positive gastric cancer. Clin Cancer Res. 2014;20:4559-73.

12. Zhao M, Luo R, Liu Y, Gao L, Fu Z, Fu Q, et al. miR-3188 regulates nasopharyngeal carcinoma proliferation and chemosensitivity through a FOXO1-modulated positive feedback loop with mTOR-p-PI3K/AKT-c-JUN. Nat Commun. 2016;7:11309.

13. Goto T, Takano M, Hirata J, Tsuda H. The involvement of FOXO1 in cytotoxic stress and drug-resistance induced by paclitaxel in ovarian cancers. Br J Cancer. 2008;98:1068-75.

14. Han CY, Cho KB, Choi HS, Han HK, Kang KW. Role of FoxO1 activation in MDR1 expression in adriamycin-resistant breast cancer cells. Carcinogenesis. 2008;29:1837-44.

15. Park J, Ko YS, Yoon J, Kim MA, Park JW, Kim WH, et al. The forkhead transcription factor FOXO1 mediates cisplatin resistance in gastric cancer cells by activating phosphoinositide 3-kinase/ Akt pathway. Gastric Cancer. 2014;17:423-30.

16. Ko YS, Cho SJ, Park J, Kim Y, Choi YJ, Pyo JS, et al. Loss of FOXO1 promotes gastric tumour growth and metastasis through upregulation of human epidermal growth factor receptor 2/neu expression. Br J Cancer. 2015;113:1186-96.

17. Kim HP, Han SW, Song SH, Jeong EG, Lee MY, Hwang D, et al. Testican-1-mediated epithelial-mesenchymal transition signaling confers acquired resistance to lapatinib in HER2-positive gastric cancer. Oncogene. 2014;33:3334-41.

18. Kim WH, Schnaper HW, Nomizu M, Yamada Y, Kleinman HK. Apoptosis in human fibrosarcoma cells is induced by a multimeric synthetic Tyr-Ile-Gly-Ser-Arg (YIGSR)-containing polypeptide from laminin. Cancer Res. 1994;54:5005-10. 
19. Kim JW, Kim HP, Im SA, Kang S, Hur HS, Yoon YK, et al. The growth inhibitory effect of lapatinib, a dual inhibitor of EGFR and HER2 tyrosine kinase, in gastric cancer cell lines. Cancer Lett. 2008;272:296-306.

20. Satoh T, Xu RH, Chung HC, Sun GP, Doi T, Xu JM, et al. Lapatinib plus paclitaxel versus paclitaxel alone in the second-line treatment of HER2-amplified advanced gastric cancer in Asian populations: TyTAN: a randomized, phase III study. J Clin Oncol. 2014;32:2039-49.

21. Sebolt-Leopold JS, English JM. Mechanisms of drug inhibition of signalling molecules. Nature. 2006;441:457-62.

22. Sharma SV, Settleman J. Oncogene addiction: setting the stage for molecularly targeted cancer therapy. Genes Dev. 2007;21: 3214-31.

23. Wu Y, Shang X, Sarkissyan M, Slamon D, Vadgama JV. FOXO1A is a target for HER2-overexpressing breast tumors. Cancer Res. 2010;70:5475-85.

24. Chakrabarty A, Bhola NE, Sutton C, Ghosh R, Kuba MG, Dave $\mathrm{B}$, et al. Trastuzumab-resistant cells rely on a HER2-PI3KFoxO-survivin axis and are sensitive to PI3K inhibitors. Cancer
Res. 2013;73:1190-200.

25. Wang YC, Morrison G, Gillihan R, Guo J, Ward RM, Fu X, et al. Different mechanisms for resistance to trastuzumab versus lapatinib in HER2-positive breast cancers: role of estrogen receptor and HER2 reactivation. Breast Cancer Res. 2011;13: R121.

26. Rusnak DW, Alligood KJ, Mullin RJ, Spehar GM, ArenasElliott C, Martin AM, et al. Assessment of epidermal growth factor receptor (EGFR, ErbB1) and HER2 (ErbB2) protein expression levels and response to lapatinib (Tykerb, GW572016) in an expanded panel of human normal and tumour cell lines. Cell Prolif. 2007;40:580-94.

27. Huang D, Duan H, Huang H, Tong X, Han Y, Ru G, et al. Cisplatin resistance in gastric cancer cells is associated with HER2 upregulation-induced epithelial-mesenchymal transition. Sci Rep. 2016;6:20502.

28. Karamouzis MV, Konstantinopoulos PA, Papavassiliou AG. Targeting MET as a strategy to overcome crosstalk-related resistance to EGFR inhibitors. Lancet Oncol. 2009;10:709-17. 